\title{
Driving style recognition using machine learning and
}

\section{smartphones [version 1; peer review: 2 approved with}

\section{reservations]}

\author{
Eilham Hakimie bin Jamal Mohd Lokman1, Vik Tor Goh (1D1, \\ Timothy Tzen Vun Yap (iD), Hu Ng(D) \\ ${ }^{1}$ Faculty of Engineering, Multimedia University, Cyberjaya, Selangor, 63100, Malaysia \\ ${ }^{2}$ Faculty of Computing and Informatics, Multimedia University, Cyberjaya, Selangor, 63100, Malaysia
}

V1 First published: $18 \mathrm{Jan} 2022$, 11:57

https://doi.org/10.12688/f1000research.73134.1

Latest published: 19 Dec 2022, 11:57

https://doi.org/10.12688/f1000research.73134.2

\section{Abstract}

Background: The lack of real-time monitoring is one of the reasons for the lack of awareness among drivers of their dangerous driving behavior. This work aims to develop a driver profiling system where a smartphone's built-in sensors are used alongside machine learning algorithms to classify different driving behaviors.

Methods: We attempt to determine the optimal combination of smartphone sensors such as accelerometer, gyroscope, and GPS in order to develop an accurate machine learning algorithm capable of identifying different driving events (e.g. turning, accelerating, or braking).

Results: In our preliminary studies, we encountered some difficulties in obtaining consistent driving events, which had the potential to add "noise" to the observations, thus reducing the accuracy of the classification. However, after some pre-processing, which included manual elimination of extraneous and erroneous events, and with the use of the Convolutional Neural Networks (CNN), we have been able to distinguish different driving events with an accuracy of about $95 \%$. Conclusions: Based on the results of preliminary studies, we have determined that proposed approach is effective in classifying different driving events, which in turn will allow us to determine driver's driving behavior.

\section{Keywords}

Machine learning, driver profiling, smartphone, convolutional neural networks
Open Peer Review

Approval Status

1

2

version 2

(revision)

19 Dec 2022

version 1

18Jan 2022

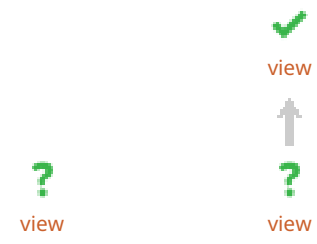

1. Muhammad Reza Z'aba, Mimos Berhad,

Kuala Lumpur, Malaysia

\section{Babul Salam KSM Kader Ibrahim (iD),}

Coventry University, Coventry, UK

Any reports and responses or comments on the article can be found at the end of the article. 


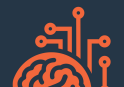

This article is included in the Artificial

Intelligence and Machine Learning gateway.

This article is included in the Research Synergy

Foundation gateway.

Corresponding author: Vik Tor Goh (vtgoh@mmu.edu.my)

Author roles: bin Jamal Mohd Lokman EH: Data Curation, Formal Analysis, Investigation, Methodology, Software, Writing - Original Draft Preparation; Goh VT: Conceptualization, Supervision, Writing - Review \& Editing; Yap TTV: Methodology, Supervision, Writing Review \& Editing; Ng H: Writing - Review \& Editing

Competing interests: No competing interests were disclosed.

Grant information: The researchers sincerely appreciate and express gratitude for financial support from the Ministry of Higher Education, Malaysia, under the Fundamental Research Grant Scheme with grant number FRGS/1/2020/TK02/MMU/02/1.

The funders had no role in study design, data collection and analysis, decision to publish, or preparation of the manuscript.

Copyright: ( 2022 bin Jamal Mohd Lokman EH et al. This is an open access article distributed under the terms of the Creative Commons Attribution License, which permits unrestricted use, distribution, and reproduction in any medium, provided the original work is properly cited.

How to cite this article: bin Jamal Mohd Lokman EH, Goh VT, Yap TTV and Ng H. Driving style recognition using machine learning and smartphones [version 1; peer review: 2 approved with reservations] F1000Research 2022, 11:57

https://doi.org/10.12688/f1000research.73134.1

First published: 18 Jan 2022, 11:57 https://doi.org/10.12688/f1000research.73134.1 


\section{Introduction}

Driver behavior strongly influences road safety ${ }^{1}$ and is currently the main contributor to traffic fatalities. Although many recorded incidents are caused by human errors, researchers suggest that drivers who exhibit a more aggressive driving style are more likely to be engaged in an accident on the $\mathrm{road}^{2}$. In 2018, a total of 548,598 cases of road accidents have been recorded in Malaysia with 6,284 of them resulting in fatalities ${ }^{3}$. To add to that, the Malaysian Institute of Road Safety Research Institute (MIROS) predicts that the number of fatalities will continue to increase up to 10,716 by the end of $2020^{4}$. Driver profiling attempts to understand and monitor the driver's behaviour in real-time, leveraging a safer and more responsible driving.

Driving style profiling is the process of collecting driving data (e.g., acceleration, braking, speed, turning rate, location) then applying them to classification models in order to generate a score to determine whether their driving style is safe or unsafe. Driver profiling has gained an increased in demand particularly in the insurance field and rental fleets. For example, AXA FlexiDrive rewards their customers with up to a $20 \%$ discount off their insurance premiums if they display good driving behaviour ${ }^{5}$. In the rental car industry, rental companies turn to RentalMatics, an IoT solution to track rental fleets in real time ${ }^{6}$. A telematics unit (also called black box) is fitted into a car to gather the relevant data needed to monitor and track the vehicle's location and behaviour ${ }^{7}$.

Today's smartphone is embedded with advanced motion sensors which is suitable for data collection. Smartphones have access to a suite of advanced sensors including accelerometer, gyroscope, magnetometer, gravity sensors and GPS. Previous works like ${ }^{8-11}$ proved that if smartphones are properly calibrated, they could be viable alternatives to the conventional telematics unit for monitoring driver behaviour.

This research aims to create a viable system to gather data through a mobile device and apply machine learning algorithms to classify different types of driving events. The research will explore the data gathering phase, where a smartphone will be used as a data collection device to replace the telematics device. Initial calibration of the motion sensors will be applied to ensure the most accurate reading of data for any vehicle. Machine learning algorithms will then be trained and tested to ensure the accuracy in determining different driving events such as careful, normal, careless, and dangerous.

\section{Methods}

This project was divided into two main parts; the first part was the data collection part which is to develop an intuitive smartphone application with the correct combination of sensors to gather raw data on basic vehicular movements and the second part is applying machine learning to train and classify different driving patterns.

\section{Data collection}

Data collection utilized the smartphone's sensors including accelerometer, gyroscope, and GPS. The accelerometer's readings
( $x, y$ and $z$ axes) relative to the phone position were recorded every $100 \mathrm{~ms}$ for the duration of the data collection. The gyroscope sensor was used to measure the vehicle's rate of rotation while the GPS was used to calculate the speed of the vehicle. Figure 1 shows the user interface of the smartphone app. The app recorded several driving event such as:

- Right turns $\left(90^{\circ}\right)$

- Left turns $\left(90^{\circ}\right)$

- Acceleration

- Braking

The driving events were attempted at a constant speed of $30 \mathrm{~km} / \mathrm{h}$. For each driving event, 30 sets of readings were collected in total.

\section{Data classification}

The classification technique employed to assess the implementation of this approach was the 1-Dimensional Convolutional Neural Network (CNN) and the CNN model was built using Tensorflow and Keras. Prior to fitting the machine learning model with data, the data was pre-processed beforehand to overcome problems such as unbalanced data or redundant data which could cause overfitting of model.

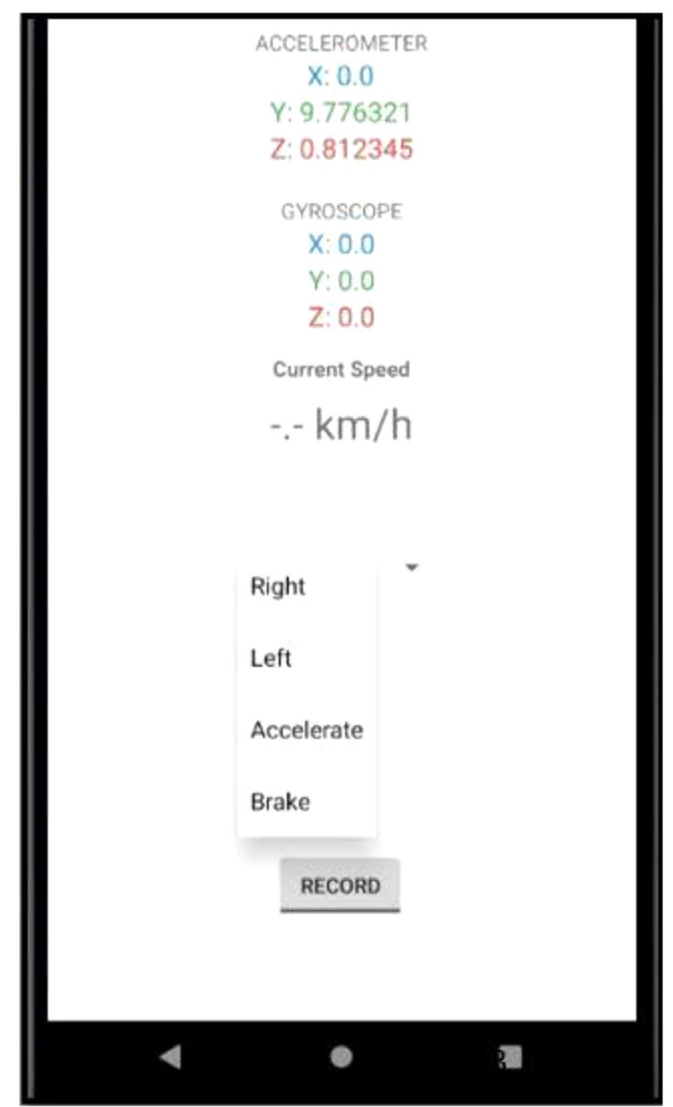

Figure 1. Screenshot of App. The figure shows the user interface of the data gathering app where readings from the smartphone's accelerometer and gyroscope are updated in real-time. 
Pre-processing began by balancing the data to ensure that the data points for all driving events are equal to prevent skewing of results. Data standardization was also implemented before model fitting. This step is vital to ensure that the model can handle lower valued numbers as the data from the sensors has fluctuations which would cause the result to be inaccurate and require more processing to compensate. Data standardization 'minimizes' the amplitude of the data into a form that is much easier to handle by the computer. Frame preparation was done during the pre-processing stage where the frame size was selected to be four seconds and the hop size was chosen to have an overlap of 24 data points. A total of 240 frames were created to accommodate the sample size. The sample data was then split into $80 \%$ for training set and $20 \%$ testing set.

After pre-processing, the CNN layer was built. The neural network layer was built following multiple trial and errors to ensure the outcome produces a good-fit model graph. An initial 1dimensional CNN layer called 'Conv1D' was created. The layer develops a convolutional kernel that convolves with the layer input over a single dimensional space to produce a tensor of outputs. Following the initial layer, an activation layer called the Rectified Linear Unit (ReLU) was applied as it is the ideal activation layer for Multilayer Perceptron (MLP) and CNN. ReLU is an activation function that utilizes simple arithmetic that will output the value directly if it is more than 0.0 , and will output 0.0 if the input is less than $0.0^{12}$. After that, a pooling layer by the name of 'MaxPool1D' was applied to reduce the number of feature maps by taking the maximum value over a certain pool size. This layer is recommended in CNN models as it reduces variance and minimizes computations ${ }^{13}$. A 'Dropout' layer is then applied which operates by setting the outgoing edges of each node to zero to minimize overfitting ${ }^{14}$. A 'Dense' layer is applied to allow the neurons in the 'Dense' layer to receive input from neurons of the previous layer ${ }^{15}$. Finally, a 'Softmax' layer is applied to convert the output into a probability distribution. Figure 2 visualizes the 1-dimension $\mathrm{CNN}$ used for this research.

\section{Results and discussion}

The readings from the smartphone's accelerometer for the various driving events are shown in Figure 3. Due to the

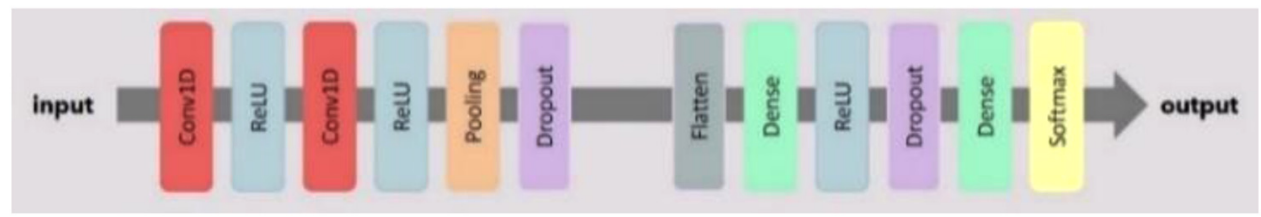

Figure 2. 1-D Convolution Neural Network. The illustration above shows the various layers used to develop the machine learning model. The various layers include Conv1D layers, Rectified Linear Unit (ReLU) Activation function, Pooling layers, Dropout, and Dense layers.

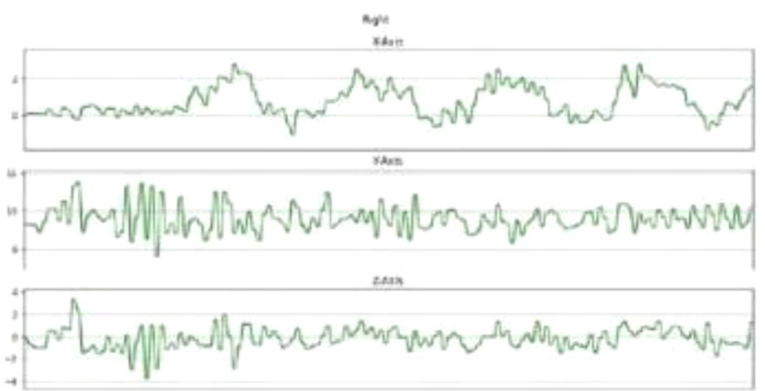

(a)

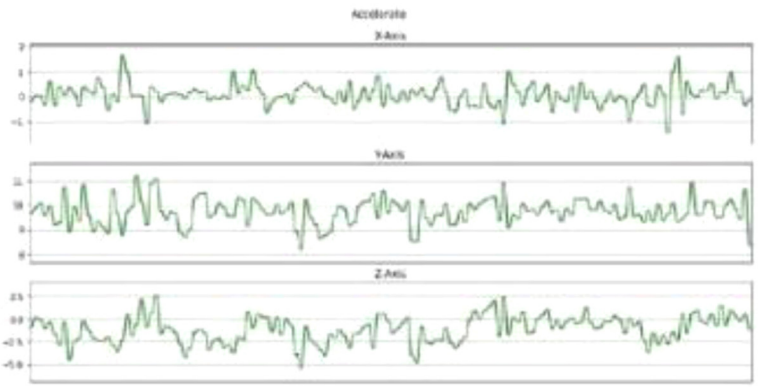

(c)

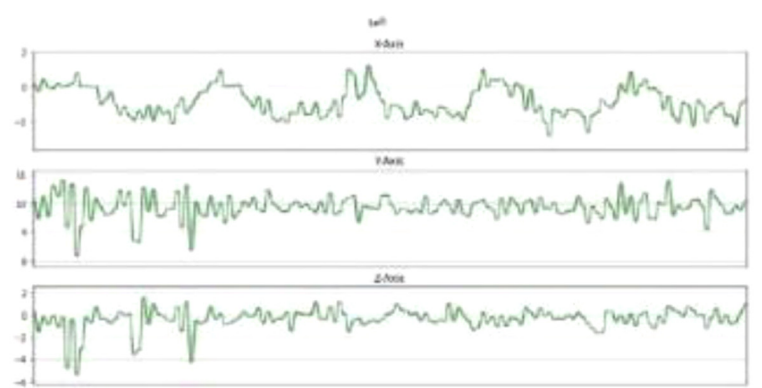

(b)

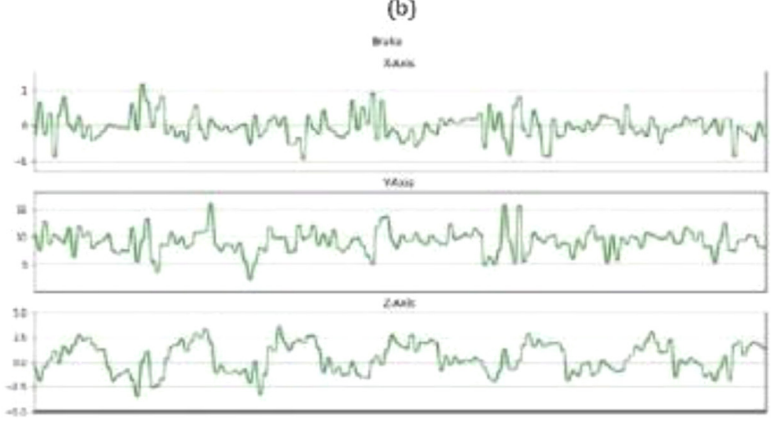

(d)

Figure 3. Accelerometer readings. (a) The $x$-axis of the accelerometer form peaks at each Right turning. (b) Left turn creates an opposite pattern from Right turns as each turning point forms successive valleys upon the $x$-axis. (c) Accelerate event creates valleys on the $z$-axis of the accelerometer. (d) Brake event indicates an opposite pattern where peaks are formed on the $z$-axis of the accelerometer at each braking point. 
vibrations of the vehicle during idling and during movements, a few spikes in the amplitude can be seen. The spikes in the accelerometer readings during idling could also be caused by the vehicle engine running.

The different types of driving events that were demonstrated could be easily distinguished between each other. In Figure 3(a), the accelerometer readings for the $x$-axis displays multiple peaks successively. Each peak in the graph translates to a right turn where the highest point of each peak translates to the highest force during each turn.

On the other hand, the readings for left turn events show the opposite result where in Figure 3(b), the accelerometer readings for the $x$-axis displays multiple valleys successively. Each valley translates to a single left turn in real life. However, Figure 3(a) and 3(b) display a close resemblance in the $y$ and $z$-axis during the early phase of the recording, where the $y$ and $z$-axis shows multiple peaks followed by multiple valleys. This could possibly be caused by the vibrations coming out from the engine of the car moving from a stationary position.

In Figure 3(c), the accelerometer values show readings for the vehicle during acceleration. A series of valleys are formed in the $z$-axis where each valley translates to an acceleration event being performed. The intensity of the valley is not very pronounced as the throttle was gradually applied to ensure smooth acceleration. If the accelerator was pressed more aggressively, we predict the $z$-axis value of the accelerometer to be more pronounced. Figure 3(d) shows the accelerometer readings for the vehicle under braking events. The output is the opposite of acceleration event, as the $z$-axis of the accelerometer form peaks at each point of braking. Each peak is much more visually defined as the brakes were applied much more aggressively to fully stop the car from $30 \mathrm{~km} / \mathrm{h}$. In both Figure $3(\mathrm{c})$ and $3(\mathrm{~d})$, the $x$ and $y$-axis produced a few peaks followed by valleys. This could be caused by the inertia acting on the vehicle or the engine vibrations in each driving event.

As observed from the accelerometer plots, each driving event could be distinguished from each other as each event has their specific features. For left and right turns there is a distinct difference in their $x$-axes while acceleration and braking events can be distinguished in the $z$-axis. The difference in features for each driving event could be highly advantageous as we could train machine learning algorithms to identify their specific features and automatically categorize each of the driving events.

The machine learning experiment was carried in two phases, training phase and test phase. $80 \%$ of the total sample data was used to train the $1-\mathrm{CNN}$ model and $20 \%$ of the sample data was used to test the accuracy of the trained model. After numerous experiments, it is discovered that the most optimal epoch value is 100 combined with a batch size of 32 which produced a model accuracy of $95.83 \%$. Figure 4 shows the test results of the machine learning model ${ }^{16}$.

The Model Loss Curve in Figure 5 shows that the training loss decreases as the epoch value increases. Upon reaching epoch value 80 , the training loss stops decreasing and stabilizes. Similarly, the validation loss decreases as the epoch value increases but stabilizes at around 80 epochs. It can be observed that the validation loss and training loss has very minimal gap between them which states that the model is a good fit model. Continued training of this model will likely cause the model to be overfitted.

Figure 6 visualizes that out of the model predicted 46 out of 48 events correctly. The two events that were wrongly predicted was an Accelerate event which was supposed to be a Left turn event and a Braking event which was supposed to be an Accelerate event. A probable reason as to why the model predicted these events wrongly is because of high variance of data because of the difficulty in maintaining consistency in simulating the driving events. Additional pre-processing of data could also improve the accuracy of the model as it removes redundant data and noise. Increasing the epoch value could also further improve the accuracy but caution must be kept in mind to make sure that the model is not overfitted.

Additional work could be done to improve the quality of data samples. For example, investing on a phone mount that does not wobble too much under engine vibrations. The data gathering framework could also be improved to ensure that only the relevant data samples are collected and not redundant data. For the application side, the sampling rate could also be tweaked to ensure that redundant accelerometer and gyroscope readings are not recorded.

\section{Conclusion}

Smartphone sensors have improved massively over the years and prove to be a viable option when used as a data gathering

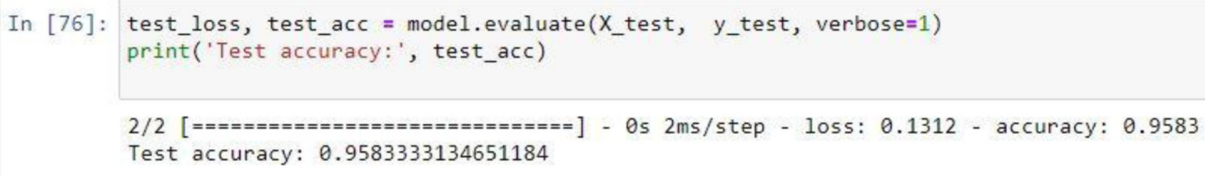

Figure 4. Model Evaluation. The figure visualizes the evaluation results for the model when classifying the testing set where an accuracy of $95.83 \%$ was obtained. 

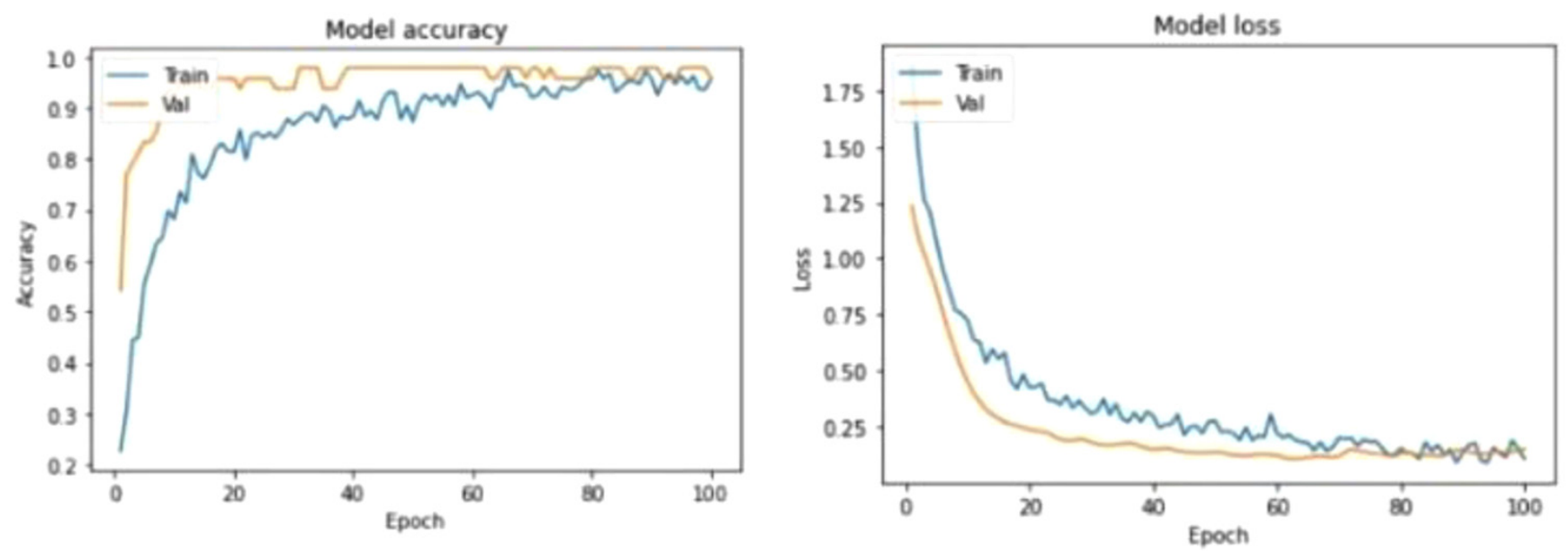

Figure 5. Learning Curves. The figure depicts the learning curves for Model Accuracy and Model Loss where each graph has a curve for Training and Validation performances.

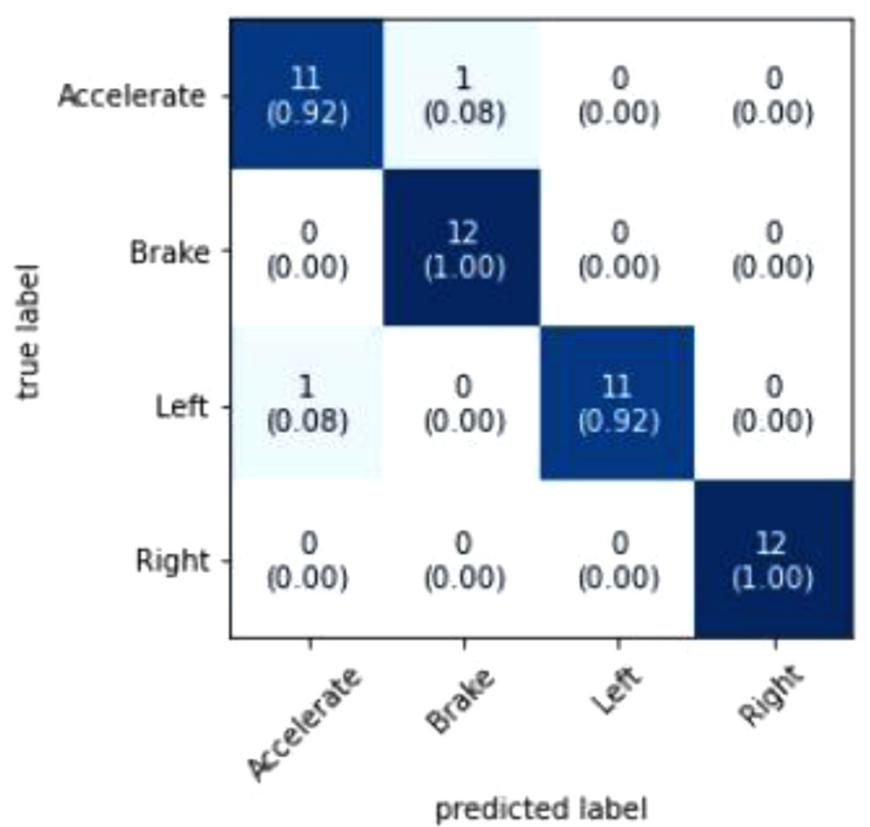

Figure 6. Confusion Matrix. The figure shows the Confusion Matrix where the prediction results are summarized to show the performance of the model.

apparatus to monitor driver behavior. In turn, the collected data could then be processed using machine learning algorithms to classify different driving events with high reliability. For this research we have applied the concept of Convolutional Neural Networks which works effectively in classifying different driving events with an accuracy of $95.83 \%$.

For our future work, we will continue to collect data for different driving events such as aggressive driving and careless driving. Additionally, we will feed the collected data into the machine learning model to allow it to classify an even more diverse palette of driving events. Next, we will consider adding a calibration feature into our data gathering application to allow accurate data collection without mounting the phone to a phone mount which will further improve user experience. As there are many machine learning models suitable for classifying time-series sensor data, we will experiment with different types of machine learning models to find out which will provide the best accuracy. Finally, we will deploy the machine learning model onto a smartphone to allow real-time classification of 
driving style. With these steps planned, it could potentially enhance the impact of this research in terms of traffic safety which was the main goal of this paper.

\section{Data Availability}

Harvard Dataverse: Driving Events, https://doi.org/10.7910/DVN/ F5JZHF' ${ }^{16}$.
This project contains the following underlying data:

- driving_events.db (Raw data from sensors)

Data are available under the terms of the Creative Commons Zero "No rights reserved" data waiver (CCO 1.0 Public domain dedication).
1. Abojaradeh $\mathrm{M}$, Jrew $\mathrm{B}, \mathrm{Al}-\mathrm{Ababsah} \mathrm{H}$, et al.: The Effect of Driver Behavior on Freeway Traffic Flow. Civ Environ Res. 2014; 6(1): 39-54.

Reference Source

2. Roslin EN, Azmy NSA, Ghulam AM, et al.: Factors of the 'aggressive driving' behaviour amongst Malaysian drivers. Int J Eng Adv Technol. 2019; 8(6): 3359-3366.

Publisher Full Text

3. D. of Statistics: Department of Statistics Malaysia. Press Release, 2015.

4. Rohayu S: Predicting Malaysian Road Fatalities for Year 2020. 2020 Reference Source

5. What You Need To Know About Telematics Motor Insurance. AXA Malaysia (accessed Dec. 14, 2020). Reference Source

6. Telematics For Rental Fleets: Shaping the Mobility Industry. RentalMatics, (accessed Dec. 14, 2020). Reference Source

7. Telematics Explained - What is Telematics? (accessed Dec. 14, 2020). Reference Source

8. Júnior JF, Carvalho E, Ferreira BV, et al.: Driver behavior profiling: An investigation with different smartphone sensors and machine learning. PLoS One. 2017; 12(4): e0174959.

PubMed Abstract | Publisher Full Text | Free Full Text
9. Fazeen M, Gozick B, Dantu R, et al.: Safe Driving Using Mobile Phones. IEEE Trans Intell Transp Syst. 2012; 13(3): 1462-1468. Publisher Full Text

10. Castignani G, Derrmann T, Frank R, et al.: Driver behavior profiling using smartphones: A low-cost platform for driver monitoring. IEEE Intell Transp Syst Mag. 2015; 7(1): 91-102. Publisher Full Text

11. Johnson DA, Trivedi MM: Driving style recognition using a smartphone as a sensor platform. IEEE Conf Intell Transp Syst Proceedings ITSC. 2011; 1609-1615. Publisher Full Text

12. A Gentle Introduction to the Rectified Linear Unit (ReLU). (accessed Jun. 22 2021).

Reference Source

13. tf.keras.layers.MaxPool1D. TensorFlow Core v2.5.0., (accessed Jun. 22, 2021). Reference Source

14. Dropout Neural Network Layer In Keras Explained. by Cory Maklin Towards Data Science, (accessed Jun. 22, 2021). Reference Source

15. Keras Dense Layer Explained for Beginners. MLK - Machine Learning Knowledge, (accessed Jun. 22, 2021). Reference Source

16. Goh VT, Lokman JM, Hakimie E, et al.: Driving Events. Harvard Dataverse, 2021. http://www.doi.org/10.7910/DVN/F5JZHF 


\section{Open Peer Review}

\section{Current Peer Review Status: ? ?}

\section{Version 1}

Reviewer Report 08 July 2022

https://doi.org/10.5256/f1000research.76760.r143359

(C) 2022 KSM Kader Ibrahim B. This is an open access peer review report distributed under the terms of the Creative Commons Attribution License, which permits unrestricted use, distribution, and reproduction in any medium, provided the original work is properly cited.

\section{Babul Salam KSM Kader Ibrahim}

Faculty of Engineering, Environment and Computing, Coventry University, Coventry, UK

This paper discusses the attempt to use the smartphone sensors such as accelerometer, gyroscope, and GPS in order to develop an accurate machine learning algorithm capable of identifying different driving events (e.g. turning, accelerating, or braking). The convolutional neural network (CNN) has been applied to classify these different events.

The title should be corrected as the work recognises the driving events, not the driving style.

The methodology should be improved by explaining and showing where the smartphone is mounted on the screen or on the dashboard. The illustration may help the reader.

Mounting on the dashboard is not a good option as there are vibrations due to the car's engine. This should be avoided.

The labels in Figure 3 are not very clear.

Please proofread the document before submission. There are too many grammatical mistakes and need to be addressed as follows-

Replace

Conclusions: Based on the results of preliminary studies, we have determined that the proposed approach is effective in classifying different driving events, which in turn will allow us to determine driver's driving behavior.

with

Conclusions: Based on the results of preliminary studies, we have determined that the proposed approach is effective in classifying different driving events, which in turn will allow us to determine driver's driving behavior. 


\section{Replace}

Driver profiling attempts to understand and monitor the driver's behaviour in real-time, leveraging a safer and more responsible driving.

with

Driver profiling attempts to understand and monitor the driver's behaviour in real-time, leveraging safer and more responsible driving.

Replace

The app recorded several driving event such as:

with

The app recorded several driving events such as:

Replace

Prior to fitting the machine learning model with data, the data was pre-processed beforehand to overcome problems such as unbalanced data or redundant data which could cause overfitting of model.

with

Prior to fitting the machine learning model with data, the data was pre-processed beforehand to overcome problems such as unbalanced data or redundant data which could cause overfitting of the model.

\section{Replace}

The neural network layer was built following multiple trial and errors to ensure the outcome produces a good-fit model graph.

with

The neural network layer was built following multiple trials and errors to ensure the outcome produces a good-fit model graph.

\section{Replace}

Figure 3. Accelerometer readings. (a) The x-axis of the accelerometer form peaks at each Right turning. (b) Left turn creates an opposite pattern from Right turns as each turning point forms successive valleys upon the $x$-axis. (c) Accelerate event creates valleys on the z-axis of the accelerometer. (d) Brake event indicates an opposite pattern where peaks are formed on the z-axis of the accelerometer at each braking point.

with

Figure 3. Accelerometer readings. (a) The $x$-axis of the accelerometer form peaks at each Right turn. (b) The Left turn creates an opposite pattern from Right turns as each turning point forms successive valleys upon the $x$-axis. (c) Accelerate event creates valleys on the $z$-axis of the accelerometer. (d) The brake event indicates an opposite pattern where peaks are formed on the z-axis of the accelerometer at each braking point.

\section{Replace}

Figure 3(a) and 3(b) display a close resemblance in the $y$ and z-axis during the early phase of the recording, where the $y$ and $z$-axis shows multiple peaks followed by multiple valleys.

with

Figures 3(a) and 3(b) display a close resemblance in the $y$ and z-axis during the early phase of the recording, where the $y$ and z-axis show multiple peaks followed by multiple valleys. 


\section{Replace}

As observed from the accelerometer plots, each driving event could be distinguished from each other as each event has their specific features.

with

As observed from the accelerometer plots, each driving event could be distinguished from the other as each event has its their specific features.

\section{Replace}

The machine learning experiment was carried in two phases, the training phase, and test phase with

The machine learning experiment was carried out in two phases, the training phase and the test phase

\section{Replace}

A probable reason as to why the model predicted these events wrongly is because of high variance of data because of the difficulty in maintaining consistency in simulating the driving events.

with

A probable reason why the model predicted these events wrongly is because of the high variance of data because of the difficulty in maintaining consistency in simulating the driving events.

\section{Replace}

It can be observed that the validation loss and training loss has very minimal gap between them which states that the model is a good fit model

with

It can be observed that the validation loss and training loss have a very minimal gap between them which states that the model is a good fit model

\section{Replace}

Figure 6 visualizes that out of the model predicted 46 out of 48 events correctly.

with

Figure 6 visualizes that the model predicted 46 out of 48 events correctly.

\section{Replace}

Next, we will consider adding a calibration feature into our data gathering application to allow accurate data collection without mounting the phone to a phone mount which will further improve user experience.

with

Next, we will consider adding a calibration feature to our data gathering application to allow accurate data collection without mounting the phone to a phone mount which will further improve the user experience.

\section{Is the work clearly and accurately presented and does it cite the current literature? Yes}

Is the study design appropriate and is the work technically sound? 
Yes

Are sufficient details of methods and analysis provided to allow replication by others? Partly

If applicable, is the statistical analysis and its interpretation appropriate?

Yes

Are all the source data underlying the results available to ensure full reproducibility?

Yes

Are the conclusions drawn adequately supported by the results?

Yes

Competing Interests: No competing interests were disclosed.

Reviewer Expertise: Sensors, Modelling and Control

I confirm that I have read this submission and believe that I have an appropriate level of expertise to confirm that it is of an acceptable scientific standard, however I have significant reservations, as outlined above.

Author Response 14 Dec 2022

Vik Tor Goh

The title will be revised to "Driving Event recognition using machine learning and smartphones" to better reflect the content of the paper. However, this is subject to the approval of F1000's editors.

The smartphone was placed on the center dashboard via a phone mount. The paper has been revised to include this information for better clarity.

We have already tested different position such as left side of the dashboard, right side of the dashboard, and even on the center console below the radio but came to a conclusion that the center of the dashboard was the best option for this car.

The labels in Figure 3 have been enhanced for better clarity.

All suggested amendments have been done. The rest of the document has also been proofread to the best of our ability to minimise grammatical and typological errors. Thank you very much for highlighting the errors.

Competing Interests: No competing interests were disclosed. 


\section{https://doi.org/10.5256/f1000research.76760.r122549}

(C) 2022 Z'aba M. This is an open access peer review report distributed under the terms of the Creative Commons Attribution License, which permits unrestricted use, distribution, and reproduction in any medium, provided the original work is properly cited.

\section{Muhammad Reza Z'aba \\ Mimos Berhad, Kuala Lumpur, Federal Territory of Kuala Lumpur, Malaysia}

The article describes an attempt to use mobile phone as an apparatus to collect data regarding certain driving events such as turning, accelerating or braking. Then, convolutional neural network (CNN) is applied to classify these different events. The authors claim that CNN managed to classify the events with a high accuracy (95.83\%).

I think the title is a bit misleading. The title mentions about driving style recognition. However, what the work recognises was the driving events such as turning, accelerating or braking. When "style" was mentioned, I was thinking something along the lines of "careless driving" or "careful driving". In essence, I believe the work has yet to recognise the driving style, only the driving events. It is good if the authors can categorise several driving styles according to a set of driving events.

The authors mention that in order to improve data collection, it is proposed to use a phone mount in order to eliminate inaccurate readings due to engine vibrations. I think the authors should do this and include the results in the article since I think a phone mount is not that expensive and is widely available. The results can then perhaps be compared with the data collected without using the phone mount. This is to see how much accuracy can be gained.

The authors also did not state the model of the smartphone used in the work. It is also good if different smartphone models are used in order to make a comparison.

Is the work clearly and accurately presented and does it cite the current literature? Partly

Is the study design appropriate and is the work technically sound? Partly

Are sufficient details of methods and analysis provided to allow replication by others? Partly

If applicable, is the statistical analysis and its interpretation appropriate? Not applicable

Are all the source data underlying the results available to ensure full reproducibility? Partly

Are the conclusions drawn adequately supported by the results? Partly 
Competing Interests: No competing interests were disclosed.

Reviewer Expertise: Information security, cryptography

I confirm that I have read this submission and believe that I have an appropriate level of expertise to confirm that it is of an acceptable scientific standard, however I have significant reservations, as outlined above.

Author Response 14 Dec 2022

Vik Tor Goh

The title will be revised to "Driving event recognition using machine learning and smartphones" to better reflect the content of the paper. However, this is subject to the approval of F1000's editors.

A phone mount was indeed used to collect data in the current experimental setup. The mount was semi-rigid as this provided a good transfer of energy/vibration from the vehicle as well as some dampening against minor vibrations. It functioned as intended but also introduced some unwanted wobble due to its semi-rigid build. As such, in our future experiments, we will investigate using other models of mounts with different build quality.

The experiments used a Samsung Galaxy S10 as the data collector. The paper has been revised to include some additional information about the data collection setup. A comparison of different smartphone models was not in the scope of the current phase but will be considered in our future experiments.

Competing Interests: No competing interests were disclosed.

The benefits of publishing with F1000Research:

- Your article is published within days, with no editorial bias

- You can publish traditional articles, null/negative results, case reports, data notes and more

- The peer review process is transparent and collaborative

- Your article is indexed in PubMed after passing peer review

- Dedicated customer support at every stage

For pre-submission enquiries, contact research@f1000.com 